\title{
A dynamically consistent discretization method for Goodwin model
}

\author{
F. Grassetti ${ }^{\mathrm{a}, *}$, M. Guzowska $^{\mathrm{b}}$, E. Michetti ${ }^{\mathrm{a}}$ \\ a Department of Economics and Law, University of Macerata, via Crescimbeni 14, Macerata 62100, Italy \\ ${ }^{\mathrm{b}}$ Institute of Econometrics and Statistics, University of Szczecin, Poland
}

\section{A R T I C L E I N F O}

\section{Article history:}

Received 30 March 2019

Revised 29 August 2019

Accepted 30 August 2019

Available online 6 September 2019

\section{Keywords:}

Goodwin model

Nonlinear dynamics

Discretization methods

Quantitative analysis

Cross-correlation

Wavelet semblance analysis

\begin{abstract}
A B S T R A C T
In economic theory the majority of macroeconomic models describing economic growth employ differential equations or sets of differential equations (see, among all, Solow, 1956 and Haavelmo, 1954). Nevertheless, economic data are usually available in discrete time. Therefore, when attempting to apply these models it is often necessary to use their discrete form, i.e. difference equations. To this aim, more and more often authors propose and analyse discrete versions of the models originally built with the assumption of time continuity. Despite many standard numeric techniques and ready-made software, obtained discrete models do not always maintain model characteristics in continuous time and the long run behaviours of the discretized model could differ from the original one. In this work, we present a modification of non-standard discretization method related to the methodology proposed by Mickens (2000), (2003) and its revisions (see Kwessi et al., 2018) for converting economic models from continuous time to discrete time. Such a discretization method preserves the original dynamic properties of the continuous model, in the sense of equilibria, their stability and bifurcation characteristics. Furthermore, the discretization produces solution trajectories in qualitative and quantitative agreement with those of the continuous model. An example of economic model described by a system of nonlinear differential equations is studied: we applied the discretization method to the Goodwin model (Goodwin, 1967) and provided a comparative analysis for qualitative and quantitative long run behaviour of the continuous and discrete version of the system.
\end{abstract}

(c) 2019 Elsevier Ltd. All rights reserved.

\section{Introduction}

The choice between continuous and discrete time to be used in the construction of dynamic models is a moot question (see Gandolfo [7]). In literature, the majority of macroeconomic models describe the economic growth by means of differential equations (ODE) or systems of such equations (see, for example, Solow [27], Swan [29], Haavelmo [11] and Ramsey [22]). Nevertheless, since most of the economic data are available in discrete time, in order to use these models in practice, it is often necessary to convert them to their discrete-time form (difference equations (DE)). Despite many standard numerical techniques and available software, the obtained discrete-time models do not always retain the properties of the original models in continuous time: different discretizations may produce complex dynamics, and even chaos, in a model system that originally lacked such dynamics. A dynamically consistent discretization scheme should produce the same type of bifurcations. For example, models originated by first-order differential equations should only produce saddle node, pitchfork, or

\footnotetext{
* Corresponding author.

E-mail address: f.grassetti@unimc.it (F. Grassetti).
}

transcritical bifurcations and should not perform period doubling bifurcation or chaos. Nevertheless, the Euler method (see Roger [24]) as well as other standard discretization schemes may produce such complex dynamics. The logistic ODE, for example, does not produce oscillations or chaotic dynamics, yet discretization methods commonly used by economists produce DE versions having damped cycles, limit cycles, or chaotic behaviour (see, among all, Day [3]). Numerous examples of discretized models with different long run behaviour exist in literature: Stutzer [30] proposed a discrete version of the Haavelmo model, Pohjola [21] discretized the Goodwin's growth cycle model while Nusse and Hommes [20] proposed a new discrete version of the Samuelson [25] model.

After the period of fascination with chaotic behaviour occurring in discrete-time models, in research fields such as biology or technical sciences the scientists started searching for methods and approaches which could be used for the discretization of models while still maintaining their dynamic properties (Mickens [19]). They developed new non-standard discretization methods such as the Kahan [13] or Mickens [18] methods. Particularly, Ronald Mickens in $[18,19]$ proposed a method for converting ordinary differential equations into discrete-time models (discretization) that have potentially important applications in applied sciences such as 
economy, population, ecology and engineering. The discretization method of Mickens in literature is referred to as the non-standard discretization formulation (NSFD). NSFD has been often used in applied mathematics to minimize the substantial distorting effects that discretization can create for ODE. For example Al-Kahby et al. [1] and Liu and Elaydi [16] applied NSFD respectively to biological and cooperative models, Roger [23] used NSFD for first order differential equations having three fixed-points, Letellier et al. [15] compared the Rössler ODE system with difference equation versions while Guzowska [10] applied NSFD methods for Goodwin model.

Here, we make discretization of the Goodwin model using the nearly exact discretization scheme (NEDS) as proposed by Kwessi et al. [14]. The main advantage of this method is its simplicity when compared to other schemes. In order to verify the efficacy of such a method, we use both topological and statistical analysis. More in detail, we show that the qualitative dynamics do not change when moving from continuous to discrete time. In addition, as regards quantitative dynamics, by considering elliptical regressions of the invariant curve as well as by performing a WaveletBased semblance analysis we concluded that the discretization is successful when the step-size, parametrized by $h$, is sufficiently low. The Wilcoxon Rank Sum test confirmed the goodness of the discretization method.

The paper is organised as follows. In Section 2 we introduce the NEDS methodology while Section 3 presents the discrete version of the Goodwin model and its qualitative dynamics. Section 4 shows the quantitative comparison between the two systems. Section 5 concludes the paper.

\section{NEDS - nearly exact discretization scheme}

In this section we introduce the Nearly Exact Discretization Scheme as proposed by Kwessi et al. [14]. NEDS method is a more general version of the NSFD method proposed by Mickens. In addition NEDS is more schematic, hence the application is easier then NSFD which is sometimes more experimental method.

Let $x$ be a quantity that changes over time. The ODE notation of $x$ is $x(t)$ whereas the DE notation for $x$ is $x_{t}$. Consider also an ODE of the form

$\frac{d x}{d t}=f(x(t))$

where $f(x)$ is a real-valued function of $x$. Then, the discrete version of (2.1) is said dynamically consistent if the conditions listed in the following definition hold.

Definition 2.1 (Kwesi et al. [14]). A discretization scheme will be called dynamically consistent if the following holds:

A1: The stability of the ODE and DE is the same.

A2: The bifurcations of the ODE and DE are the same.

A3: If two ODEs are equivalent through change of parametrization, then the resulting DEs must be equivalent through the same parametrization.

According to the previous definition, a discretization scheme is dynamically consistent when the long run behaviour of the discrete model (meant as its attractors and bifurcations) replies that of the continuous one. Note that a discretization scheme may be dynamically consistent but does not still perform equal dynamics in transition states. A discretization methodology that takes in consideration also the behaviour in transition states is introduced in the definition below.

Definition 2.2 (Kwesi et al. [14]). A discretization scheme is called a Nearly Exact Discretization Scheme if it is dynamically consistent and the trajectories of the resulting DE are the same or nearly the same as those of ODE.
A NEDS of the ODE in (2.1) can be obtained using the following algorithm:

$P 1)$ The derivative $\frac{d x}{d t}$ can be discretized as

$$
\frac{x_{t+1}-x_{t}}{\phi(h)}
$$

where $\phi$ depends on a step size $h$ and other parameters, and is given as

$$
\phi(h)=h^{2}+O\left(h^{2}\right) \text { as } h \rightarrow 0^{+} .
$$

P2) If the right hand side of (2.1) is of the form $f(x)=r x \pm$ $g(x) x$, where $r$ is a non-zero real constant, it is assumed

$\phi(h)=\frac{e^{r h}-1}{r}$

from which

$$
\frac{x_{t+1}-x_{t}}{\phi(h)}=\left\{\begin{array}{l}
r x_{t}+g\left(x_{t}\right) x_{t+1} \\
r x_{t}-g\left(x_{t}\right) x_{t+1}
\end{array}\right.
$$

and the resulting DE is given by

$$
x_{t+1}=f_{0}\left(x_{t}\right)=\left\{\begin{array}{l}
\frac{e^{r h} x_{t}}{1-\phi(h) g\left(x_{t}\right)} \\
\frac{e^{r h} x_{t}}{1+\phi(h) g\left(x_{t}\right)}
\end{array}\right.
$$

P3) If the right hand side of (2.1) is of the form $f(x)=r x \pm$ $g(x) x+b$, then

$$
x_{t+1}=f_{0}\left(x_{t}\right)=\left\{\begin{array}{l}
\frac{e^{r h} x_{t}+\phi(h) b}{1-\phi(h) g\left(x_{t}\right)} \\
\frac{e^{r h} x_{t}+\phi(h) b}{1+\phi(h) g\left(x_{t}\right)}
\end{array} .\right.
$$

Notice that, due to its simplicity, NEDS can be used for other economic models. This non standard discretization scheme can be used in many types of difference equations and also in systems of difference equations (see Kwessi [14]).

In the next section, the introduced algorithm will be applied to the Goodwin model. We underline that our application leads to a system behaving in a way which has not been considered in Kwessi [14]. Our aim is to provide a dynamically consistent discretization of the continuous system and to compare their dynamics both from a qualitative and quantitative point of view.

\section{From continuous to discrete dynamics - the model}

Goodwin growth cycle model [8] was a milestone in working on non-linear dynamic systems in economics. Since then, many improvements and discussion have been introduced (see Desai [4], Gandolfo [7], Goodwin [9] and Wolfstetter [31]). The Goodwin model can be represented as follows (Desai et al.[5]; Sordi and Vercelli $[28])$

$\left\{\begin{array}{l}\frac{d v}{d t}=[1 / \sigma-(\alpha+\beta)-(1 / \sigma) u] v \\ \frac{d u}{d t}=[-(\alpha+\gamma)+\rho v] u\end{array}\right.$

where numerical constants $\alpha, \beta, \gamma, \sigma$ and $\rho$ are considered to be positive $^{1}, v$ is the employment rate and $u$ is the wage share. Assuming that $a=\frac{1}{\sigma}-(\alpha+\beta), b=\frac{1}{\sigma}, c=\alpha+\gamma, d=\rho$ the continuous form of the model (3.1) can be written as

$$
C G:\left\{\begin{array}{l}
\dot{v}(t)=a v(t)-b u(t) v(t) \\
\dot{u}(t)=-c u(t)+d u(t) v(t)
\end{array} .\right.
$$

\footnotetext{
${ }^{1}$ Parameters $\alpha$ and $\beta$ are growth rates respectively of labour productivity and labour supply, $\sigma$ is the capital/output ratio while $\gamma$ and $\rho$ are related to the variation of real wage rate under assumption of linear approximation.
} 

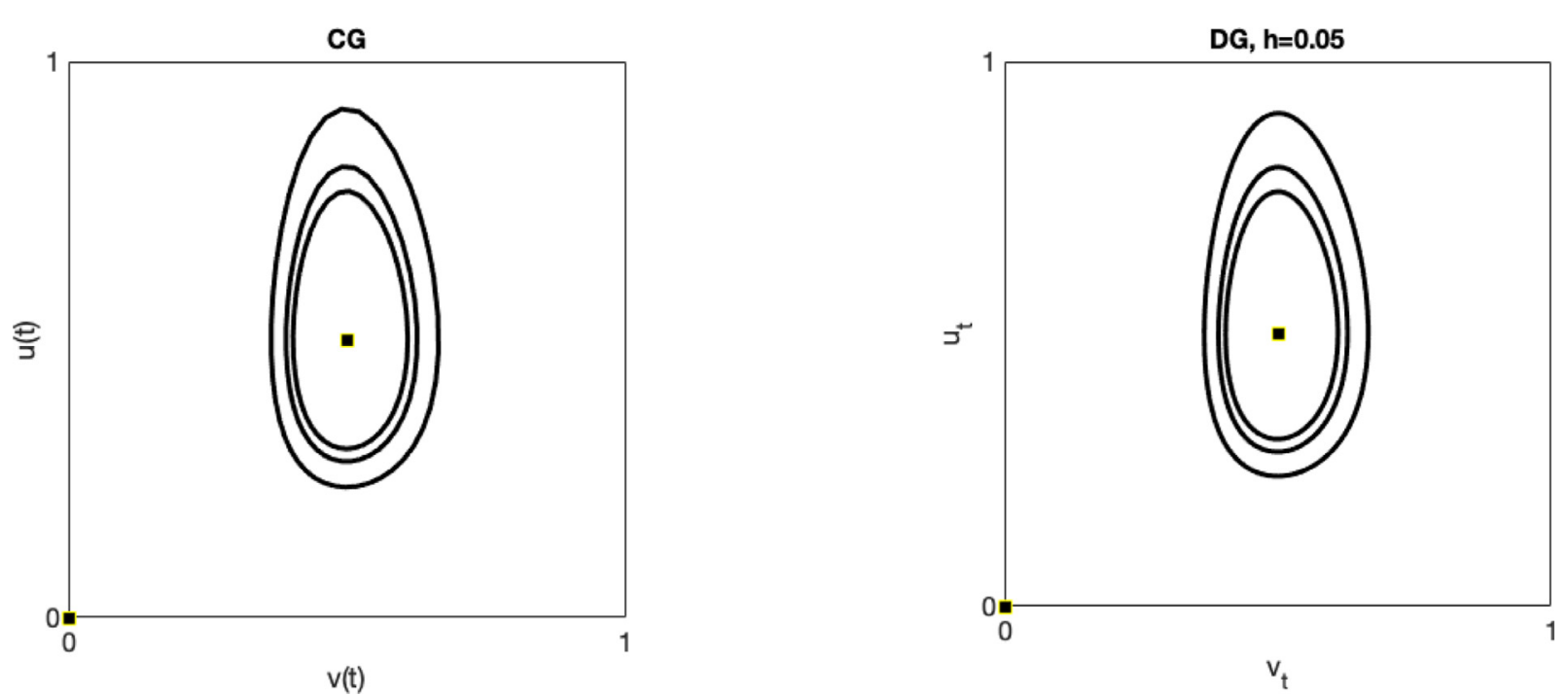

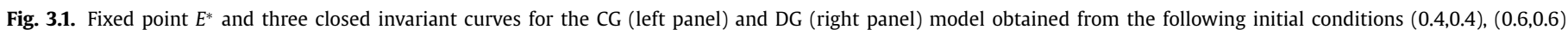
and $(0.65,0.65)$ showing that $E^{*}$ is a stable centre for both models.
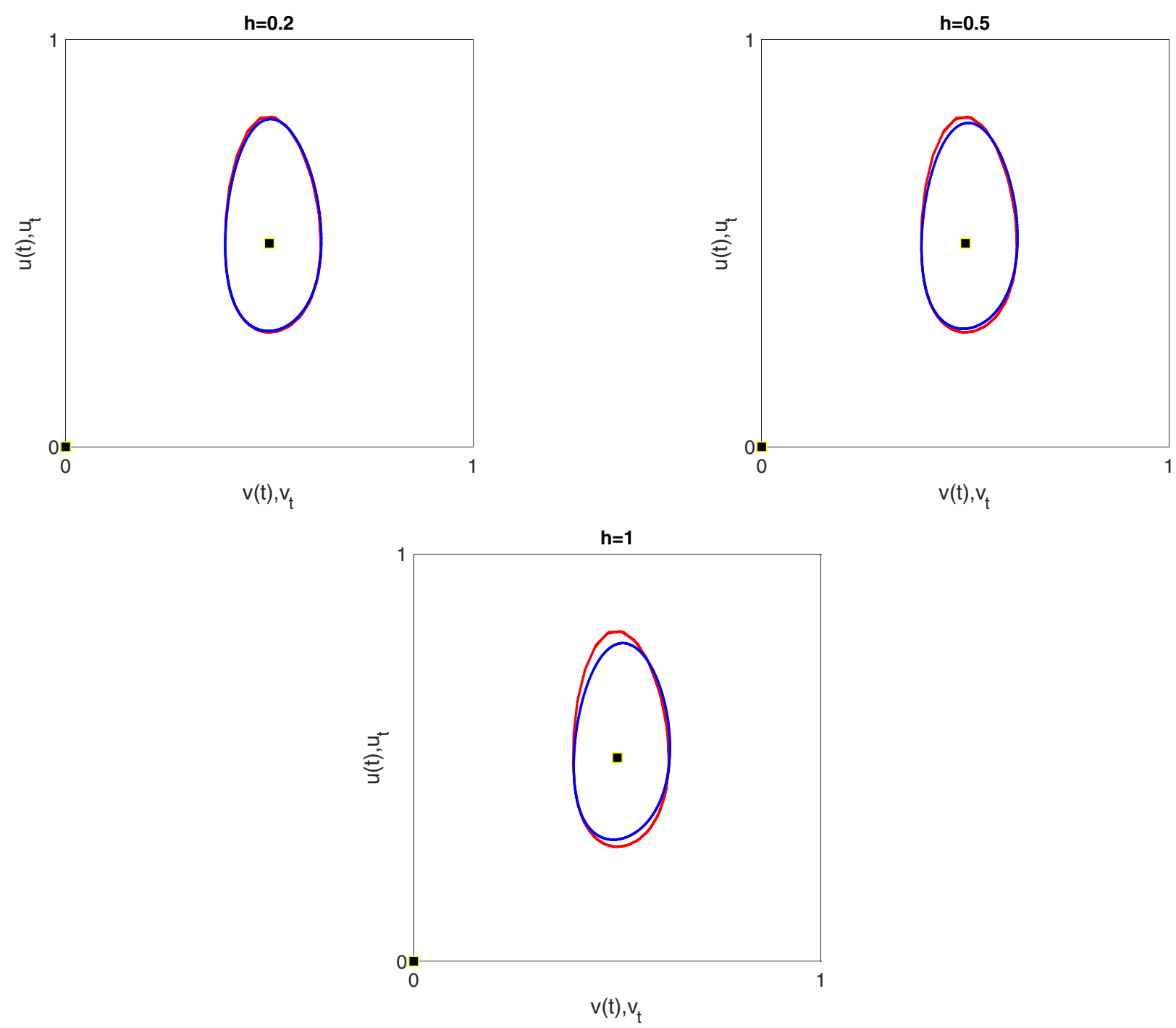

Fig. 4.1. Comparison between continuous (red) and discrete (blue) models for $a=.1, b=.2, c=.5$ and $d=1$ for different values of $h$ and the same initial condition. 

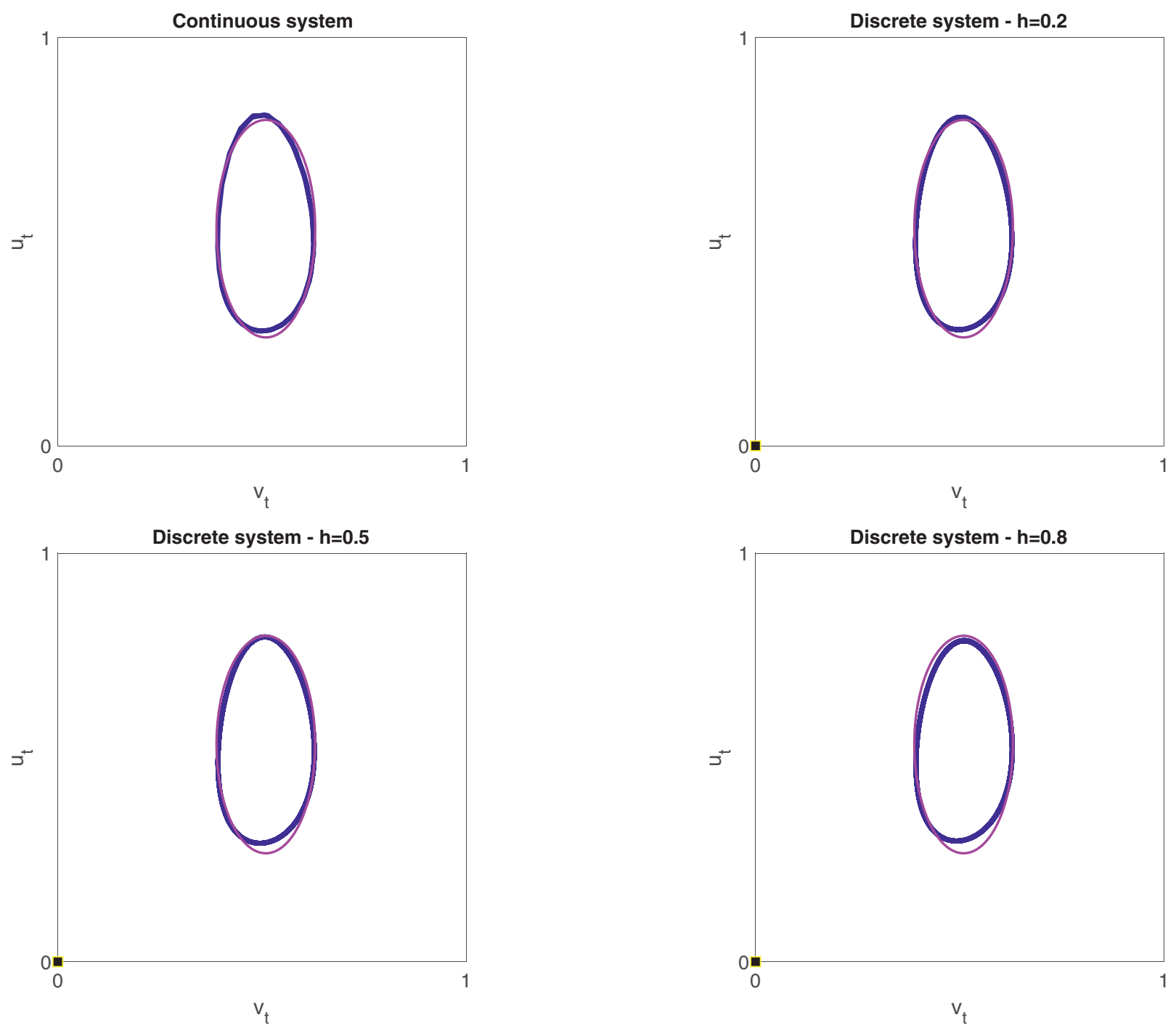

Fig. 4.2. Points belonging to $Z$ (in blue) and estimated ellipse (in magenta) for $a=.1, b=.2, c=.5$ and $d=1$.

Fixed points and local stability of the continuous time Goodwin model have been widely studied (see Gandolfo [7] for a complete description of the continuous time two-dimensional Goodwin model). We briefly recall such results. System (CG) has two equilibrium points: the origin $E_{0}=(0,0)$ and an interior fixed point $E^{*}=\left(v^{*}, u^{*}\right)=\left(\frac{c}{d}, \frac{a}{b}\right)$.

The dynamic properties of the model (CG) can be easily demonstrated. The Jacobian matrix of the model is:

$J_{0}(v, u)=\left[\begin{array}{cc}a-b u & -b v \\ d u & -c+d v\end{array}\right]$.

When evaluated at the steady state $E_{0}=(0,0)$ the Jacobian matrix becomes:

$J_{0}(0,0)=\left[\begin{array}{cc}a & 0 \\ 0 & -c\end{array}\right]$.

Point $E_{0}=(0,0)$ is a hyperbolic saddle point, because $\operatorname{Det}\left(J_{0}(0,0)\right)=-a c<0$. For the point $E^{*}=\left(v^{*}, u^{*}\right)=\left(\frac{c}{d}, \frac{a}{b}\right)$ the Jacobian matrix is:

$J_{0}\left(v^{*}, u^{*}\right)=\left[\begin{array}{cc}0 & -\frac{b c}{d} \\ \frac{a d}{b} & 0\end{array}\right]$.
As the eigenvalues are both purely imaginary $\left(\lambda_{1}=-i \sqrt{a c}, \lambda_{2}=\right.$ $i \sqrt{a c})$ this fixed point is not hyperbolic, so no conclusions can be drawn from the linear analysis. However, the continuous model CG behaves as the Lotka-Volterra two-dimensional predator-pray model, where it is shown that $E^{*}$ is a centre (see Liu and Elaydi [16]). After recalling the qualitative properties of the continuous model, we now apply the NEDS method to system CG as given by (3.2) in order to provide a discrete version of the Goodwin model that preserves the behaviour of the original one. By applying the algorithm proposed in previous section, we obtain the difference equation system:

$D G:\left\{\begin{array}{l}v_{t+1}=\frac{v_{t}+a \Phi v_{t}}{1+b \Phi u_{t}} \\ u_{t+1}=\left(1-c \Psi+d \Psi \frac{v_{t}+a \Phi v_{t}}{1+b \Phi u_{t}}\right) u_{t}\end{array}\right.$,

where $\Phi(a, h)=\frac{e^{a h}-1}{a}$ and $\Psi(c, h)=\frac{1-e^{-c h}}{c}$.

In the following Proposition fixed points and their local stability for the continuous time (CG) and the discrete time (DG) systems are compared. 


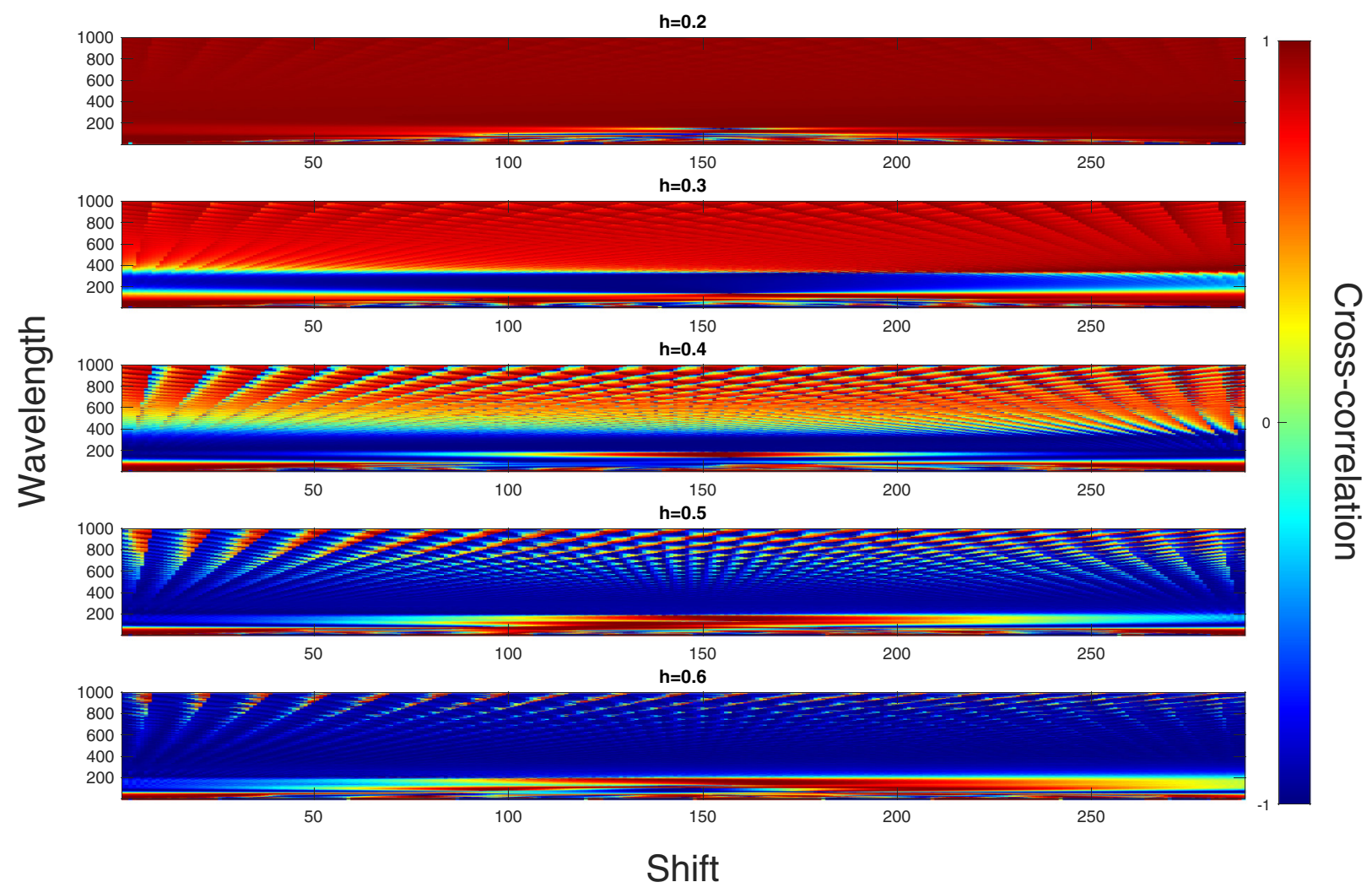

Fig. 4.3. Wavelet-based semblance analysis for $a=.1, b=.2, c=.5, d=1$ and different values of $h$.

\section{Proposition 3.1.}

(i) The discrete time model given in (3.3) has the same fixed points as the continuous time model given in (3.1).

(ii) The local stability properties of the fixed point $E_{0}=(0,0)$ of system CG is preserved for the discrete system DG.

(iii) Necessary conditions for $E^{*}$ to be a centre for system DG as for system CG hold.

\section{Proof.}

(i) Trivially, system (DG) has two equilibrium points: the origin $E_{0}=(0,0)$ and an interior fixed point $E^{*}=\left(v^{*}, u^{*}\right)=\left(\frac{c}{d}, \frac{a}{b}\right)$.

(ii) The Jacobian matrix associated to DG is:

$J_{1}(v, u)=\left[\begin{array}{cc}\frac{1+a \Phi}{1+b u \Phi} & \frac{-b \Phi(v+a v \Phi)}{(1+b u \Phi)^{2}} \\ j_{21} & j_{22}\end{array}\right]$

where $j_{21}=\frac{d u(1+a \Phi) \Psi}{1+b u \Phi}$ and $j_{22}=1-c \Psi-\frac{b d u \Phi(v+a v \Phi) \Psi}{(1+b u \Phi)^{2}}+$ $\frac{d(v+a v \Phi) \Psi}{1+b u \Phi}$.

For the point $E_{0}=(0,0)$ the Jacobian matrix becomes:

$J_{1}(0,0)=\left[\begin{array}{cc}1+a \Phi & 0 \\ 0 & 1-c \Psi\end{array}\right]$

and its eigenvalues are $\lambda_{1}=1-c \Psi$ and $\lambda_{2}=1+a \Phi$. Since $0<\lambda_{1}<1<\lambda_{2}$ it follows that $E_{0}=(0,0)$ is a saddle point.

(iii) For the point $E^{*}=\left(v^{*}, u^{*}\right)=\left(\frac{c}{d}, \frac{a}{b}\right)$ the Jacobian matrix takes the form:

$J_{1}\left(v^{*}, u^{*}\right)=\left[\begin{array}{cc}1 & -\frac{b c \Phi}{d+a d \Phi} \\ \frac{a d \Psi}{b} & \frac{1+a \Phi-a c \Phi \Psi}{1+a \Phi}\end{array}\right]$ and $J_{1}\left(v^{*}, u^{*}\right)$ has two complex conjugate eigenvalues $\lambda_{1}$ and $\lambda_{2}$, being

$$
\lambda_{1}=\frac{2+2 a \Phi-a c \Phi \Psi-\sqrt{a} \sqrt{c} \sqrt{\Phi} \sqrt{\Psi} \sqrt{-4-4 a \Phi+a c \Phi \Psi}}{2(1+a \Phi)}
$$

and

$$
\lambda_{2}=\frac{2+2 a \Phi-a c \Phi \Psi+\sqrt{a} \sqrt{c} \sqrt{\Phi} \sqrt{\Psi} \sqrt{-4-4 a \Phi+a c \Phi \Psi}}{2(1+a \Phi)},
$$

whose modulus equal one, i.e. $E^{*}$ is a not hyperbolic fixed point. Being $E^{*}$ not hyperbolic, we observe that, for all parameter values,

(i) $1+\operatorname{Tr}\left(J_{1}\left(u^{*}, v^{*}\right)\right)+\operatorname{Det}\left(J_{1}\left(u^{*}, v^{*}\right)\right)>0$,

(ii) $1-\operatorname{Tr}\left(J_{1}\left(u^{*}, v^{*}\right)\right)+\operatorname{Det}\left(J_{1}\left(u^{*}, v^{*}\right)\right)>0$,

(iii) $\operatorname{Det}\left(J_{1}\left(u^{*}, v^{*}\right)\right)=1$,

thus implying that no bifurcation occurs since the local stability of $E^{*}$ does not change when one or more parameters are moved. As the third condition for the local stability of $E^{*}$ is always violated, then $E^{*}$ can be a centre (see Medio and Lines [17] for a more in deep discussion).

Since we deal with a non-hyperbolic case, stability cannot be stated by looking only at the linear part. Due to the complex analytical form, a proof based on higher order terms cannot be obtained. Anyway, by means of numerical simulations, we verified that $E^{*}$ is a stable centre (in Fig. 3.1 three different invariant curves for CG and DG models are presented).

Our results aim at showing that the economic meaningful interior fixed point $E^{*}$ maintains its local stability properties when moving from continuous time to discrete time: in both cases $E^{*}$ is 

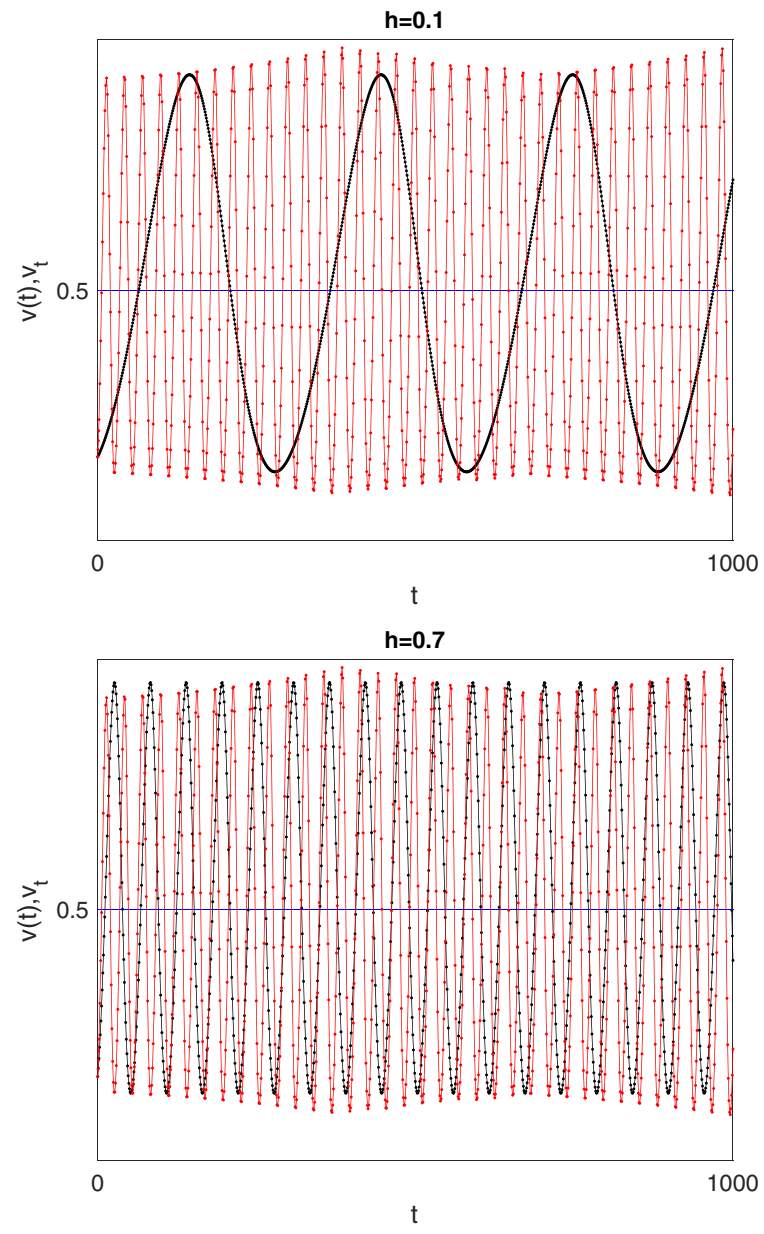
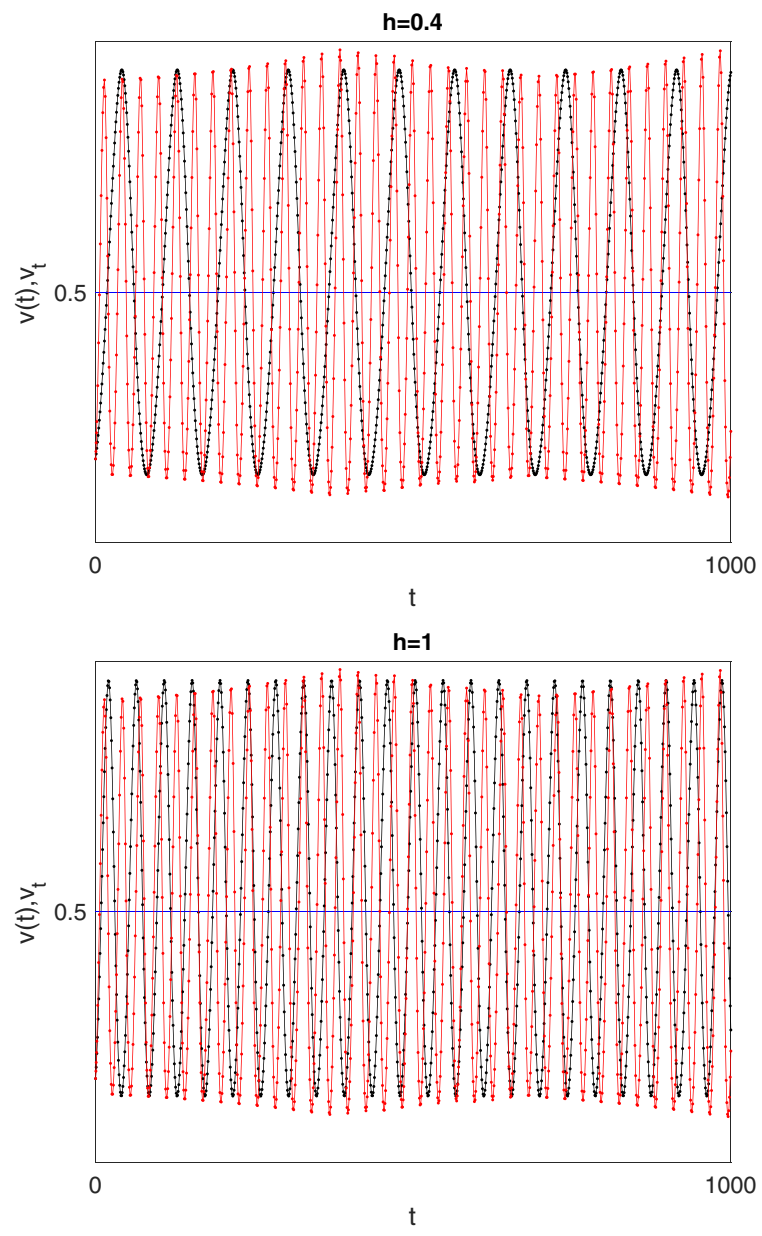

Fig. 4.4. Time series of the discrete (black) and continuous (red) model for $a=.1, b=.2, c=.5, d=1$ and different values of $h$. Fixed point in blue.

a stable centre and any initial condition produces a trajectory belonging to a closed invariant curve around $E^{*}$.

Although the qualitative behaviour of the two systems coincides, a quantitative analysis has to be done in order to verify the numerical correspondence between the two systems.

\section{Quantitative comparisons between dynamics}

The discretization method previously introduced bases its significance on the property of letting unchanged the qualitative and quantitative dynamics of the model so that it can be applied when only discrete observations are available for a continuous process. In previous section the qualitative correspondence between the continuous and discrete version of the model has been discussed. A quantitative correspondence is easily traceable when comparing the behaviour of the two systems for different values of the parameter that describes the step-size of the discretization method. We start our numerical analysis by considering the long term dynamics produced by the two systems, for given initial conditions. Fig. 4.1 shows the high resemblance on the shape of the two centres It is worthwhile to highlight that the correspondence between centres is negatively correlated with the amplitude of the step-size $h^{2}$.

In the following, we compare the topology of the discrete system's dynamical regimes to the one of the original system. Our

\footnotetext{
2 The invariant curve in continuous time has been computed with MATLAB by using the ode45 algorithm that solves non-stiff differential equations with medium order method (for details see Shampine and Reichelt [26]).
}

aim is to verify in which measure the discrete system provides the same quantitative dynamics when compared to the continuous counterpart. To this purpose, we use statistical tools and topological analysis.

\subsection{Elliptic regression}

Firstly, we use the Last-Squares criterion in order to estimate the best fit to an ellipse of the set of points $(u, v)$ which belongs to the centre of the continuous time model, then we compare the estimated parameters with those obtained by using the set of points belonging to the invariant curve of the discrete time system, for different values of the step-size parameter $h$ and the same initial condition.

Assume $\quad Z=\left\{\left(u_{t}, v_{t}\right) \in \mathbb{R}_{0}^{+2}: \exists t \geq t_{0}, t \in \mathbb{N}\right.$ and $\left(u_{t_{0}}, v_{t_{0}}\right) \in Z$ so that $\left.\left(u_{t}, v_{t}\right)=D G^{t}\left(u_{t_{0}}, v_{t_{0}}\right)\right\}$ is a closed invariant curve of the DG model given by (3.3), for given parameter values and let $u_{c}$ and $v_{c}$ be the vectors respectively of all the values $u_{t}$ and $v_{t}$ belonging to $Z$, both with length $n$. In order to fit the best ellipse given the points $(u, v) \in Z$ we use the fit-estimation method of Least Squares without weights as proposed by Gal [6]: to estimate the conic equation of the ellipse, i.e. $A u^{2}+B u v+C v^{2}+D u+E v=F$, we extract the estimator from

$g(u, v, \tilde{A}):=A u^{2}+B u v+C v^{2}+D u+E v=F \quad u, v \in Z$

where $\tilde{A}=(A, B, C, D, E)$ is a $\mathbb{R}^{5}$ vector of parameters to be estimated (notice that parameters are normalised by $-F$ ). Let $g_{c}$ be the vector function of all the measurement, i.e. the vector obtained applying function $g$ to each point belonging to $Z$, then the Squared 


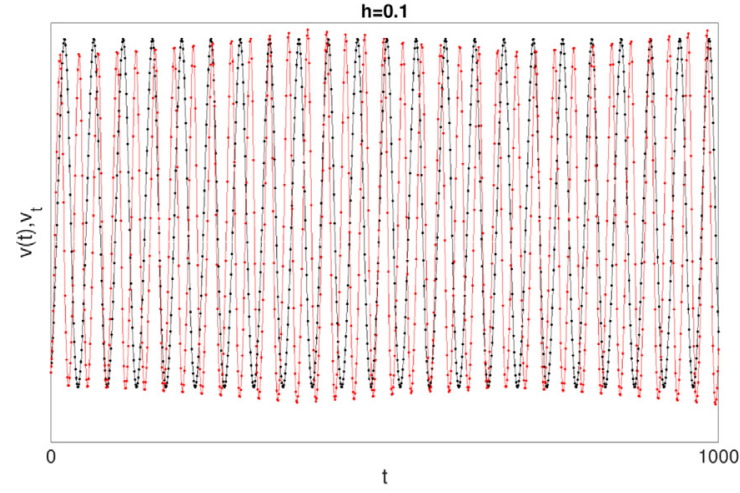

$\mathrm{h}=0.7$

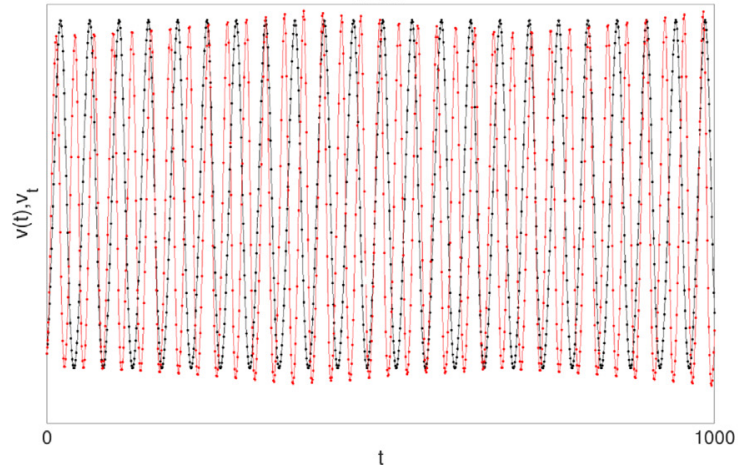

$\mathrm{h}=0.4$

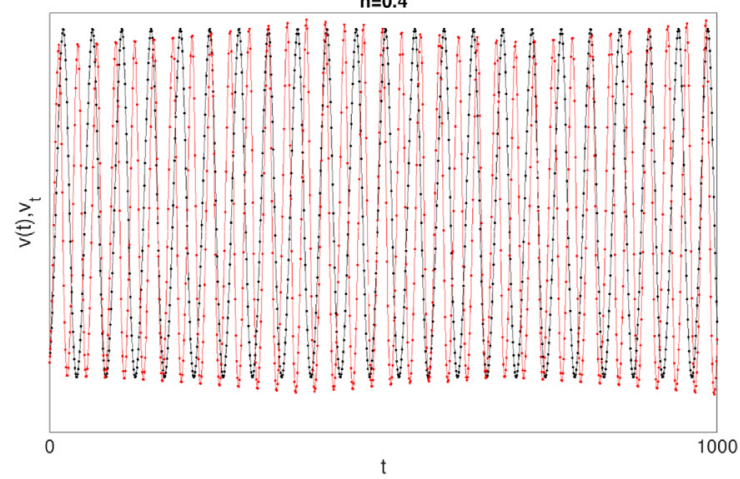

$\mathrm{h}=1$

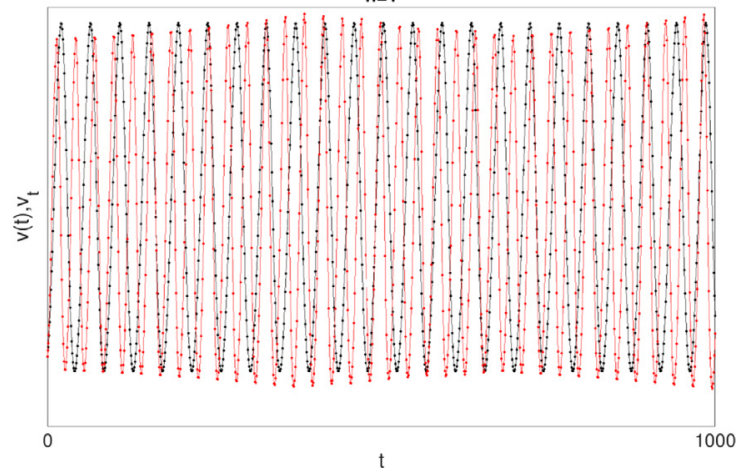

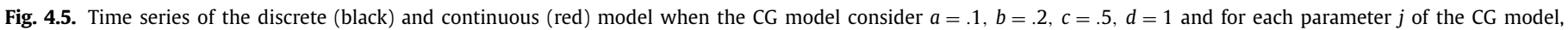
the DG model follows $j / h$.

Table 1

Sub-axis and coordinates of the centres for estimated ellipses for $a=.1, b=.2, c=.5$ and $d=1$.

\begin{tabular}{llllll}
\hline Model & $h$ value & $v$ & $\eta$ & $u_{0}$ & $v_{O}$ \\
\hline CG & - & 0.1208 & 0.2664 & 0.5095 & 0.5318 \\
DG & $\mathrm{h}=0.1000$ & 0.1195 & 0.2624 & 0.5032 & 0.5382 \\
DG & $\mathrm{h}=0.2000$ & 0.1190 & 0.2605 & 0.5014 & 0.5397 \\
DG & $\mathrm{h}=0.3000$ & 0.1188 & 0.2582 & 0.4977 & 0.5430 \\
DG & $\mathrm{h}=0.4000$ & 0.1188 & 0.2555 & 0.4946 & 0.5458 \\
DG & $\mathrm{h}=0.5000$ & 0.1187 & 0.2531 & 0.4915 & 0.5485 \\
DG & $\mathrm{h}=0.6000$ & 0.1186 & 0.2508 & 0.4885 & 0.5511 \\
DG & $\mathrm{h}=0.7000$ & 0.1185 & 0.2486 & 0.4856 & 0.5536 \\
DG & $\mathrm{h}=0.8000$ & 0.1184 & 0.2464 & 0.4825 & 0.5562 \\
DG & $\mathrm{h}=0.9000$ & 0.1183 & 0.2442 & 0.4796 & 0.5586 \\
DG & $\mathrm{h}=1.0000$ & 0.1182 & 0.2420 & 0.4762 & 0.5614 \\
\hline
\end{tabular}

Error Function is defined as

$\operatorname{SEF}(\tilde{A}):=\left(g_{c}-f_{c}\right)^{\top}\left(g_{c}-f_{c}\right)=\left(X \tilde{A}+f_{c}\right)^{\top}\left(X \tilde{A}+f_{c}\right)$

where $f_{c}$ is the vector of values $F$ obtained by applying function (4.1) to each couple $(u, v) \in Z$ while $X=\left(x_{i j}\right) \in \mathbb{R}^{n, 5}$ is defined as $X:=\left(\begin{array}{lllll}u_{c}^{2} & u_{c} \cdot v_{c} & v_{c}^{2} & u_{c} & v_{c}\end{array}\right)$. Derivation of $\operatorname{SEF}(\tilde{A})$ with respect to $\tilde{A}$ yields the estimator

$\tilde{A}_{S E}=\frac{X_{S}}{X^{\top} X}$

where $X_{S}=\left(\begin{array}{lllll}\sum_{i=1}^{n} x_{i, 1} & \sum_{i=1}^{n} x_{i, 2} & \sum_{i=1}^{n} x_{i, 3} & \sum_{i=1}^{n} x_{i, 4} & \sum_{i=1}^{n} x_{i, 5}\end{array}\right)$ is a $\mathbb{R}^{5}$ vector. The same algorithm has been applied to estimate the equation of the ellipse fitting the continuous version of the model, after selecting points belonging to the closed invariant curve obtained from the same initial condition. Sub axis $v$ and $\eta$ and centre $O\left(u_{0}, v_{0}\right)$ of each ellipse are listed in Table 1.

A graphical comparison between the points belonging to the centre and the estimated ellipse is given in Fig. 4.2. Given the
Table 2

Success of the discretization method for $\mu=0.05, a=.1, b=$ $.2, c=.5, d=1$ and initial condition $(.4, .4)$.

\begin{tabular}{llllllll}
\hline$h$ value & $C 1$ & $C 2$ & $S D$ & $h$ value & $C 1$ & $C 2$ & $S D$ \\
\hline 0.1 & $*$ & $*$ & $*$ & 0.6 & - & $*$ & - \\
0.2 & $*$ & $*$ & $*$ & 0.7 & - & $*$ & - \\
0.3 & $*$ & $*$ & $*$ & 0.8 & - & $*$ & - \\
0.4 & - & $*$ & - & 0.9 & - & - & - \\
0.5 & - & $*$ & - & 1.0 & - & - & - \\
\hline
\end{tabular}

* = fulfilled.

$-=$ not fulfilled.

equations of the ellipse fitting the points of the set $Z$ when varying $h$, we now compare the centres obtained with the continuous and discrete version of the model. We assume the discretization method successfully preserves the invariant curve of the continuous model (and we label this case as Successful Discretization (SD)) when the following conditions hold:

(i) $O_{d} \in I\left(O_{c}, \mu \eta_{c}\right)$

(ii) $\left|v_{d}-v_{c}\right| \leq \mu^{2}$

where the subscripts $c$ and $d$ refer to the ellipse estimated respectively form the continuous and discrete version of the model while $\mu$ may be considered as a degree of tolerance. In the following we will refer to conditions $(i)$ and (ii) respectively as $C 1$ and $C 2$. The results of our analysis for $\mu=0.05$ are listed in Table 2 .

Numerous simulations show that the discretization method is successful for sufficiently low values of parameter $h$.

\subsection{Wavelet-Based semblance analysis}

To complete our analysis we also compare the final outcome of the continuous model with the one obtained when considering the 
Table 3

Wilcoxon Rank Sum test. Results: $0=$ failure to reject the null hypothesis at the $5 \%$ significance level, $1=$ rejection of the null hypothesis. Parameter values: $a=.1, b=.2, c=.5, d=1$.

\begin{tabular}{llllll}
\hline & Variable $v$ & & Variable $u$ \\
$h$ value & p-value & Result & & p-value & Result \\
\hline .2 & 0.914638512670177 & 0 & 0.688223062677938 & 0 \\
.4 & 0.721938152129168 & 0 & & 0.655845171494252 & 0 \\
.6 & 0.791341257630410 & 0 & & 0.791341257630410 & 0 \\
.8 & 0.838323269321723 & 0 & & 0.838323269321723 & 0 \\
.9 & 0.926251986419469 & 0 & & 0.926251986419469 & 0 \\
\hline
\end{tabular}

discrete system, for different values of the parameter $h$. Following Cooper and Cowan [2], wavelets are used to perform semblance analysis in order to display correlations as a function of wavelength and time-shift.

Fig. 4.3 shows semblance analysis for values of $u$ belonging to the invariant curve of the continuous and discrete model. The points in the plot represent the correlation values between dataset depending on the scale and time shift. Anti-correlation is displayed in blue, zero correlation in green, and positive correlation in red. The semblance is calculated using the continuous wavelet transform. This approach takes into account the temporal variability in the spectral character of the time-series. Once again, numerous tests prove that the discrete invariant curve quantitatively matches the continuous one when the step-size $h$ is small enough. It is worthwhile to highlight that, as $h$ increases, a positive crosscorrelation holds for sufficiently high values of the wavelength. This phenomenon may be easily verified by observing the time series generated from the two systems.

Fig. 4.4 shows the wavelength of the time series generated by the discrete model, for different values of the step-size. Particularly, the phase of the dataset approaches the one of the continuous model as $h$ increases. Conversely, for smaller values of $h$, the wavelet analysis shows similarities between the two curves when a longer wave is considered for the discrete model.

Notice that, as far as prediction is concerned, the discrete model does not follow the original one when $h$ is sufficiently small. This is due to the calibration of parameters: moving the step size $h$, the temporal meaning of parameters $a, b, c$ and $d$ have to be adjusted in order to predict the exact evolution over time. Although the importance of this issue would need the attention of a new work, numerous simulations show that, for each parameter $j$ of the CG model, by assuming for the DG model that each parameter is defined $j / h$, the step size of the two models coincide (see Fig. 4.5). To conclude our analysis we perform the Wilcoxon Rank Sum test (see Hettmansperger and McKean [12]), a non parametric test with null hypothesis that the two datasets have equal medians. In Table 3 the results are listed: for all values of the step-size the test failed to reject the null hypothesis at the $5 \%$ significance level. Although this test only concerns the medians of the datasets for the two variables, it gives indications about the quantitative differences between the quantitative dynamics of the two systems, confirming the goodness of the discretization method.

\section{Conclusion}

In this paper, we present the modification of the Mickens $[18,19]$ non standard discretization method proposed by Kwesi et al. [14] and their application to the Goodwin model. The discretization algorithm preserves the qualitative and quantitative dynamics of the continuous system and may be used to convert economic models from continuous time to discrete time, when measured data are discrete. We applied the discretization method to the Goodwin model [9]. The resulting discrete system guarantees the same qualitative dynamics of the original models of any choice of the parameter values. A quantitative study carried out by means of Elliptical Regression, Wilcoxon Rank Sum test and Wavelet-Based Semblance Analysis show that the two systems have a similar quantitative behaviours and that the discretization method can be considered quantitative successful.

\section{Declaration of Competing Interest}

The project is financed within the framework of the program of the Minister of Science and Higher Education under the name "Regional Excellence Initiative" in the years 2019-2022, project number 001/RID/2018/19, the amount of financing PLN 10,684,000. 00 .

\section{References}

[1] Al Kahby H, Dannan F, Elaydi S. A non-standard discretization methods for same biological models. Applications of nonstandard finite difference schem. Mickens RE, editor. Word Scientific; 2000.

[2] Cooper GRJ, Cowan DR. Comparing time series using wavelet based semblance analysis. Comput Geosci 2008;34(2):95-102.

[3] Day RH. Irregular growth cycles. Am Econ Rev 1982;72:406-14.

[4] Desai M. Growth cycles and inflation in a model of class struggle. J Econ Theory 1973;6:527-45.

[5] Desai M, Henry B, Mosley A, Pemberton M. A clarification of the Goodwin model of the growth cycle. J Econ Dyn Control 2006;30:2661-70.

[6] Gal O. Fit ellipse. 2003. https://bit.ly/2H9rXTW.

[7] Gandolfo G. Economic Dynamics. Berlin: Springer; 1997

[8] Goodwin RM. A growth cycle. Socialism, Capitalism, and Economic Growth Feldstein CH, editor. Cambridge: Essays Presented to Maurice Dobb; 1967.

[9] Goodwin RM. Schumpeter, keynes and the theory of economic evolution. Evol Econ 1991;1:29-47.

[10] Guzowska M. Dynamiczne wlasnosci dyskretnej wersji modelu wzrostu goodwina. Zeszyty Naukowe/Uniwersytet Ekonomiczny w Poznaniu 2011;211:125-36.

[11] Haavelmo T. A study in the theory of economic evolution. Amsterdam: North-Holland; 1954.

[12] Hettmansperger TP, McKean JW. Robust nonparametric statistical methods. New York: John Wiley and Sons; 1998.

[13] Kahan W. Unconventional numerical methods for trajectory calculations. Unpublished lecture notes. Berkeley: University of California Berkley; 1993.

[14] Kwessi E, Elaydi S, Dennis B, Livadiotis G. Nearly exact discretization of single species population models. Nat Resour Model 2018;31(4):e12167.

[15] Letellier C, Elaydi S, Aguirre LA, Alaoui A. Difference equation versus differential equations, a possible equivalence for the Rössler system? Physica D 2004; 195:29-49.

[16] Liu P, Elaydi S. Discrete competitive and cooperative models of lotka-volterra type. J Comput Anal Appl 2001;3(1):53-73.

[17] Medio A, Lines M. Nonlinear dynamics: a premier. Cambridge: Cambridge University Press; 2001.

[18] Mickens RE. Nonstandard finite difference methods of differential equations World Scientific; 1993.

[19] Mickens RE. Applications of nonstandard finite difference schem. Word Scientific; 2000.

[20] Nusse HE, Hommes $\mathrm{CH}$. Resolution of chaos with application to a modified Samuelson model. J Econ Dyn Control 1990;14:1-19.

[21] Pohjola MJ. Stable and chaotic growth: the dynamics of a discrete version of Goodwin's growth cycle model. Zeitschrift für Nationalökonomie 1981:41:27-38.

[22] Ramsey FP. A mathematical theory of saving. Econ J 1928;38(152):543-59.

[23] Roeger LIW, Mickens RE. Exact finite-difference schemes for first order differential equations having three distinct fixed-points. J Diff Equ Appl 2007;13(12):1179-85.

[24] Roger LW. Local stability of Euler's and Kahan's methods. J Differ Equ Appl 2006;10(6):601-14.

[25] Samuelson PA. Interactions between the multiplier analysis and principle of acceleration. Rev Econ Stat 1939;21:75-8

[26] Shampine LF, Reichelt MW. The MATLAB ODE suite. SIAM J Sci Comput 1997; 18:1-22.

[27] Solow R. A contribution to the theory of economic growth. Q J Econ 1956;70(1):65-94.

[28] Sordi S, Vercelli A. Unemployment, income distribution and debt-financed in vestment in a growth cycle model. J Econ Dyn Control 2014;48:325-48.

[29] Swan TW. Economic growth and capital accumulation. Econ Rec 1956;32:334-61.

[30] Stutzer MJ. Chaotic dynamics and bifurcation in a macro model. J Econ Dyn Control 1980;2:353-76.

[31] Wolfstetter E. Fiscal policy and the classical growth. Zeitschrift für Nationalökonomie 1992;42:375-93. 